\title{
Stress and long-term memory retrieval: a systematic review
}

\author{
Cadu Klier, ${ }^{1} \mathbb{D}$ Luciano Grüdtner Buratto ${ }^{1} \mathbb{D}$
}

\begin{abstract}
Introduction: The experience of stressful events can alter brain structures involved in memory encoding, storage and retrieval. Here we review experimental research assessing the impact of the stress-related hormone cortisol on long-term memory retrieval.

Method: A comprehensive literature search was conducted on PubMed, Web of Science and PsycNet databases with the following terms: "stress," "Iong-term memory," and "retrieval." Studies were included in the review if they tested samples of healthy human participants, with at least one control group, and with the onset of the stress intervention occurring after the encoding phase and shortly (up to one hour) before the final memory test.

Results: Thirteen studies were included in the qualitative synthesis $(\mathrm{N}=962)$ and were classified according to the time elapsed between stress induction and memory retrieval (stress-retrieval delay), the stress-inducing protocol (stressor), the time of day in which stress induction took place, sex, and age of participants. Most studies induced stress with the Trier Social Stress Test (TSST) between 15 and 25 minutes before the final memory (mostly recall) test and showed significant increases in cortisol levels and memory impairment.

Discussion: The reviewed studies indicate that stress does impair retrieval, particularly when induced with the TSST, in the afternoon, up to 45 minutes before the onset of the final memory test, in healthy young men. These results may inform future research on the impact of stress-induced cortisol surges on memory retrieval.
\end{abstract}

Keywords: Long-term memory, memory retrieval, stress, cortisol.

\section{Introduction}

Stressful events are common in everyone's life, inducing physiological changes, such as increased heart rate and sweating. ${ }^{1}$ Stressful events can also impact memory and reasoning. Due to their relevance, stress and its main hormone, cortisol, are widely investigated. ${ }^{2-7}$ Here we review studies on the effects of stress on memory retrieval, highlighting inconsistencies found in the literature and presenting suggestions for future studies.

\section{Stress response and memory retrieval}

Stressful events activate both the sympathetic nervous system (SNS), leading to the release of noradrenaline and adrenaline (NA) into the bloodstream by the adrenal medulla, and also the hypothalamicpituitary-adrenal (HPA) axis, leading to the secretion of glucocorticoids (cortisol) into the blood by the adrenal cortex. ${ }^{3}$ Stress-induced cortisol release can have a direct impact on the hippocampus and the amygdala, brain structures involved in memory and emotional processes. ${ }^{8}$ Cortisol can cross the blood-

\footnotetext{
${ }^{1}$ Departamento de Processos Psicológicos Básicos, Instituto de Psicologia, Universidade de Brasília, Brasília, DF, Brazil. Submitted Aug 14 2019, accepted for publication Jan 302020.

Suggested citation: Klier C, Buratto LG. Stress and long-term memory retrieval: a systematic review. Trends Psychiatry Psychother. $2020 ; 42(3): 284-291$. http://dx.doi.org/10.1590/2237-6089-2019-0077
} 
brain barrier and bind to glucocorticoid receptors in the hippocampus, thus modulating hippocampal function, and consequently, modulating encoding and retrieval of long-term memories. ${ }^{9}$

A considerable amount of research has investigated the impact of stress-related cortisol release on memory processing. Empirical evidence, however, has been inconclusive, with studies showing that episodic memory can be enhanced, ${ }^{10,11}$ impaired $^{12-15}$ or remain unaffected $^{16-18}$ after a stressful event depending on the timing of the event. When the stressor occurs just before the retrieval of consolidated information, memory performance is impaired, ${ }^{7}$ as in the case of the student who knows the subject but cannot remember its contents during a high-stakes test.

\section{Present study}

In this study, we reviewed the literature published over the last 12 years on the impact of stress on memory retrieval, focusing on some key aspects, such as the time elapsed between stress induction and memory retrieval (stress-retrieval delay) and the protocol used to induce stress in the laboratory (stressor).

Stress-retrieval delay is crucial for understanding how stress modulates memory. ${ }^{15}$ The fast stress response occurs seconds after the stressor and involves the release of adrenaline, which increases alertness and facilitates memory encoding. ${ }^{6}$ The slow stress response, conversely, occurs several minutes after the stressor and involves the release of cortisol, which impairs the retrieval of consolidated memories. ${ }^{6}$ In fact, a recent metaanalysis has concluded that acute stress shortly prior to retrieval can significantly impair memory retrieval. ${ }^{7}$ Because stressful events prior to memory retrieval are fairly common (e.g., go blank during a speech), we restricted our systematic review to studies in which stress induction occurred shortly before retrieval.

Stressor type is also important, as some tasks are more effective than others at stimulating cortisol release and emphasize different aspects of the stress response (physiological vs. social). In one common protocol, the Cold Pressor Test (CPT), participants submerge their dominant hand into cold water for a short period of time. ${ }^{19}$ The cold water triggers the HPA axis, inducing the release of cortisol. ${ }^{9}$ By contrast, in the Trier Social Stress Test (TSST), the participant takes part in a task that includes giving a public speech and doing mental arithmetic aloud. ${ }^{20}$ While stress induction in the CPT is driven mainly by physiology, in the TSST stress induction is influenced by social evaluation and unpredictability. ${ }^{21}$ Given these differences in the nature of the stressors, we ask whether their impact on memory retrieval may also be different.
In addition to timing and stressor, we also classified studies according to the time of day in which the retrieval session took place (morning vs. afternoon), sex, and age of participants. These factors were deemed important because they are known to affect cortisol response and because previous studies reported conflicting results. For example, cortisol levels are high in the morning and lower in the afternoon. ${ }^{22}$ However, stress-related memory impairment appears not to vary with time of day. ${ }^{23}$ Also, stress-related memory impairment seems to be sexand gender-specific, having been observed mostly in young male participants ${ }^{16}$ : older participants are less affected, likely due to their lower responsiveness to circulating cortisol levels, ${ }^{24}$ and women are less affected depending on the phase of their menstrual cycle. ${ }^{18,25} \mathrm{We}$ classified the search results along these factors (e.g., sex, age) in order to further explore their moderating influence on stress-related memory deficits.

\section{Method}

The systematic review was conducted following the Preferred Reporting Items for Systematic Reviews and Meta-Analyses (PRISMA). 26,27 PRISMA guidelines informed our search strategy, selection criteria, data extraction, and data analyses.

\section{Literature search}

A comprehensive literature search was conducted by the first author in January 2020 on three databases: Web of Science, PsycNet, and PubMed. We looked for articles published from January 2008 to December 2019. On the Web of Science and PsycNet databases, the following keywords and Boolean terms were used: ("stress" AND "long-term memory" AND "retrieval"). The search on the Web of Science database was conducted on SCI-EXPANDED, SSCI, A\&HCI, CPCI-S, CPCI-SSH, and ESCI indexes. On PubMed, the search was conducted with the following Medical Subject Headings (MeSH terms): ("Stress, Psychological" OR "Stress, Physiological" OR "Stress") AND ("Memory, Long-Term" OR "Long-term memory") AND ("Mental Recall" OR "Retrieval").

\section{Inclusion and exclusion criteria}

The following inclusion criteria were taken into consideration: experimental studies published in English, from 2008 to 2019, with samples comprised of healthy human participants, with at least one control group, and with the onset of the stressor intervention occurring after the encoding phase and shortly (up to one hour) before the final memory 
test. Literature reviews, experimental studies with animals or with human patients (e.g., chronic stress disorders), studies without stress manipulation or with stress manipulation occurring at a time other than before the final memory test (e.g., before or during encoding) were excluded.

\section{Data extraction and management}

Eligible studies were selected based on title and abstract screening. Data from eligible studies were individually extracted and recorded in separate databases by the first author. Uncertainties were resolved through discussion with the second author. To improve objectivity, data from each study were organized through standardized forms containing the following categories: First author, year of publication, number of participants, participants' age and sex, stressor, stressretrieval delay, physiological measurement tools (e.g., cortisol saliva sampling), type of stimuli (e.g., word pairs, images, text), and type of final memory test (e.g., free recall).

\section{Results}

The initial search conducted on the three databases plus previously known articles yielded a total of 273 results: Web of Science $(n=125)$, PsycNet $(n=42)$, PubMed ( $n=99)$, and other sources ( $n=7)$. After removing duplicates, a total of 181 articles were screened (title and abstract) to check their relevance for this review. During the initial screening, 161 articles were excluded following the exclusion criteria. A total of 20 articles were assessed in full for eligibility. During fulltext review, the first assessment was to check whether stress induction occurred before the final memory test. Five studies were excluded because stress onset occurred at a different moment of the experiment (e.g., during encoding or consolidation), and two studies were excluded because they tested cognitive functions other than long-term memory. Even though the literature on the impact of stress on memory performance is vast, only 13 studied survived our strict search criteria, with a total of 962 participants. Figure 1 shows the PRISMA flow diagram for study selection.

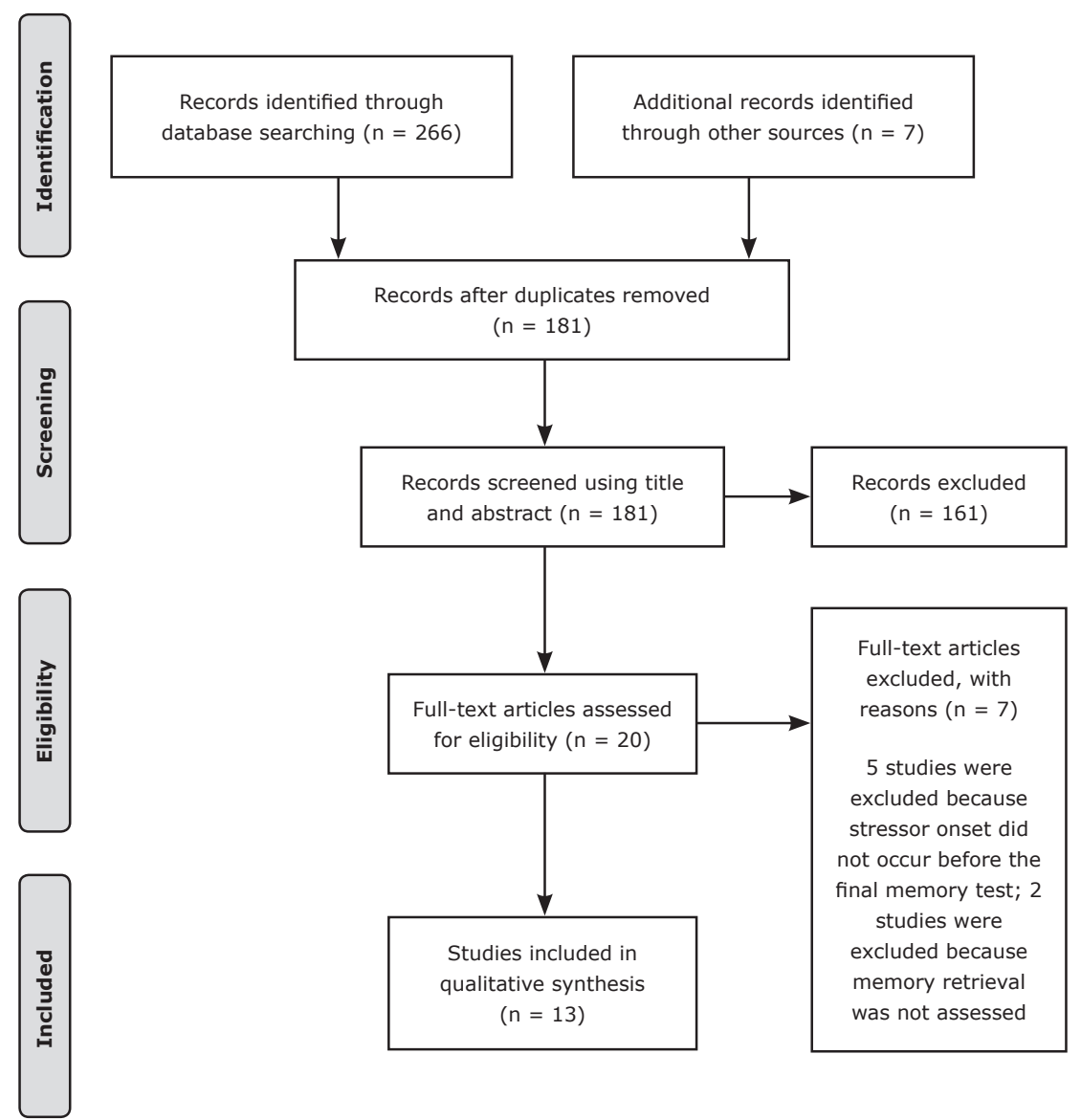

Figure 1 - Systematic review flow diagram in accordance with the Preferred Reporting Items for Systematic Reviews and Meta-Analyses (PRISMA). 


\section{Systematic review findings}

Studies differed in several methodological dimensions, namely, types of stimuli (pictures, words, non-word syllable, text passage, search task and word-pairs), stressors (described below), stressretrieval delay, time of day in which the retrieval session took place (morning vs. afternoon), sex, and age of participants (young vs. old participants). Table 1 summarizes the search results. A total of 25 experimental conditions (hereafter "experiments") were described in the 13 reviewed studies. Eighteen out of the 25 experiments reviewed showed that stress affects memory retrieval. Data from two of these experiments showed memory enhancement, whereas data from the remaining 16 experiments showed memory impairment for participants in the stress group or for those classified as stress responders in a post-hoc analysis.

\section{Stressors}

Three stress induction protocols were identified in the search results, namely, the CPT, the Socially Evaluated Cold Pressor Test (SECPT) and the TSST. In the CPT, participants are told to submerge their dominant hand into cold water $\left(0-3^{\circ} \mathrm{C}\right)$ for a short period of time (1-3 $\mathrm{min}$ ). The CPT triggers the HPA axis, leading to the release of cortisol. ${ }^{9}$ In the control task, participants submerge their dominant hand into warm water (around $30^{\circ} \mathrm{C}$ ) for the same amount of time. Four studies used CPT as the stress inducing protocol, ${ }^{10,11,13,28}$ with a total of six experiments and 246 participants (111 males, 135 females).

As in the CPT, participants in the SECPT also submerge their dominant hand into cold water. Unlike the CPT, however, participants in the SECPT are videotaped by the experimenter, who informs the participant that his/her reactions to cold water will be recorded for future analysis. ${ }^{31}$ Only two studies used SECPT as a stressor, ${ }^{15,23}$ with a total of five experiments and 196 participants (94 males, 102 females).

In the TSST, participants take part in a role playing task that simulates a job interview. ${ }^{20}$ They are told to prepare a 5-minute speech to be read in front of unfamiliar people. Following the speech, participants are also told to carry out mental subtractions aloud, being interrupted by the experimenters in case of mistakes. In the control task, participants perform similar activities but rather than giving a public speech, they write a note about a friend's job interview and rather than doing mental subtractions aloud, they write the subtractions in a piece of paper. A total of seven studies used TSST as a stressor, ${ }^{12,14,16-18,29,30,32}$ with a total of 14 experiments and 520 participants (254 males, 266 females).

Reported cortisol levels varied across studies, being lower for older compared to younger participants, and lower for women compared to men. The two studies that tested older participants ${ }^{16,17}$ induced stress via the TSST. Despite smaller cortisol responsivity, older participants may experience higher levels of subjective stress in psychosocial tasks, such as the TSST. ${ }^{9}$

Some studies reported a post-hoc analysis whereby participants were grouped as responders (showing an increase of at least $2.5 \mathrm{nmol} / \mathrm{l}$ in cortisol levels) and non-responders (see Table 1, last column). The aim was to assess whether cortisol responsivity (regardless of initial assignment to control vs. stress groups) was associated with memory impairment or enhancement. Two out of the five studies that performed this analysis found an impairment in delayed recall for responders after stress induction with TSST ${ }^{14,30}$; one study reported memory enhancement after stress induction with the $\mathrm{CPT}^{11}$; and the remaining two studies reported no difference between control and stress conditions after stress induction with the TSST. ${ }^{18,29}$

\section{Stress-retrieval delay}

The time elapsed between stressor onset and the final memory test affected memory performance. In five experiments, memory was tested immediately after the stressor, and in 20 experiments there was a delay (Table 2). When the criterial test occurred immediately after the stressor, participants in the stress and control groups produced similar results, except for one study ${ }^{10}$ that reported a significant memory enhancement for men. Another study ${ }^{11}$ showed, in a post-hoc analysis, that cortisol non-responders also had their memories enhanced. When the criterial test occurred 10-45 minutes after the stressor, results varied with either no change or an impairment in memory performance (Table 2 ).

\section{Age}

Only two studies addressed age as a factor, including older participants (56-76 years of age ${ }^{16,17}$ ). Both studies reported similar memory performance for both men and women under both conditions (stress or control). The level of cortisol increase in older participants was smaller than in younger participants, which could account for the lack of stress-related memory impairment in the old age groups.

\section{Sex}

Only three studies reported significant differences in performance in the final memory test between men and women. ${ }^{10,11,16}$ When both sexes were in groups with successfully increased levels of cortisol, young men's scores on the final memory test were lower than young women's. Women in the luteal phase of the menstrual cycle are less responsive to surges in cortisol levels 
Table 1 - Studies included in the systematic review

\begin{tabular}{|c|c|c|c|c|c|c|c|c|c|c|c|c|c|c|}
\hline \multirow[b]{3}{*}{ Study } & \multirow{2}{*}{\multicolumn{3}{|c|}{$\begin{array}{l}\text { Participant } \\
\text { information }\end{array}$}} & \multirow{3}{*}{$\begin{array}{l}\text { Stimuli } \\
\text { used in } \\
\text { encoding } \\
\text { sessions }\end{array}$} & \multirow[b]{3}{*}{$\begin{array}{c}\text { Retention } \\
\text { interval }\end{array}$} & \multicolumn{4}{|c|}{ Stress protocol } & \multirow{3}{*}{$\begin{array}{l}\text { Memory test } \\
\text { type (time } \\
\text { after stress } \\
\text { onset) }\end{array}$} & \multicolumn{4}{|c|}{ Memory test results } \\
\hline & & & & & & \multirow{2}{*}{$\begin{array}{l}\text { Type of } \\
\text { stressor } \\
\text { (details) }\end{array}$} & \multirow{2}{*}{$\begin{array}{l}\text { Time of } \\
\text { retrieval } \\
\text { session }\end{array}$} & \multicolumn{2}{|c|}{$\begin{array}{c}\text { Change in } \\
\text { cortisol } \\
\text { levels } \\
\text { (peak in } \\
\text { min after } \\
\text { onset) } \\
\end{array}$} & & \multicolumn{2}{|c|}{$\mathbf{C G} \times \mathbf{S G}$} & \multicolumn{2}{|c|}{$\mathbf{R D} \times \mathbf{N R}$} \\
\hline & $\mathbf{M}$ & $F^{A}$ & $\begin{array}{c}\text { Age range } \\
\text { (mean) }\end{array}$ & & & & & $\mathbf{M}$ & $\mathbf{F}$ & & $\mathbf{M}$ & $\mathbf{F}$ & $\mathbf{M}$ & $\mathbf{F}$ \\
\hline Boehringer $^{12}$ & 51 & - & $\begin{array}{l}19-31 \\
(24.6)\end{array}$ & 30 nouns & $135 \mathrm{~min}$ & TSST & 4-6 p.m. & $-\overline{(25)}$ & $-(-)$ & $\begin{array}{l}\text { Free recall } \\
(30 \mathrm{~min})\end{array}$ & $\begin{array}{c}\text { SG } \\
\text { impaired* }\end{array}$ & - & - & - \\
\hline Goldfarb $^{13}(1)$ & 30 & 30 & $\begin{array}{l}18-35 \\
(23.0)\end{array}$ & $\begin{array}{c}\text { Multicued } \\
\text { search task } \\
\text { ("T" and "L") }\end{array}$ & $24 \mathrm{~h}$ & $\begin{array}{l}\text { CPT }\left(0-2{ }^{\circ} \mathrm{C}\right. \\
\text { for } 3 \mathrm{~min})\end{array}$ & $\begin{array}{l}\text { Noon-6 } \\
\text { p.m. }\end{array}$ & $\stackrel{-}{(15)}$ & $\stackrel{-}{(15)}$ & $\begin{array}{l}\text { Search, } \\
\text { stimulus- } \\
\text { response } \\
(15 \mathrm{~min})\end{array}$ & Same & Same & - & - \\
\hline Goldfarb $^{13}(2)$ & 30 & 30 & $\begin{array}{l}18-35 \\
(23.0)\end{array}$ & $\begin{array}{l}\text { Multicued } \\
\text { search task } \\
(\text { "T" and " } \mathrm{L} \text { ") }\end{array}$ & $24 \mathrm{~h}$ & $\begin{array}{l}\text { CPT }\left(0-2{ }^{\circ} \mathrm{C}\right. \\
\text { for } 3 \mathrm{~min})\end{array}$ & $\begin{array}{l}\text { Noon-6 } \\
\text { p.m. }\end{array}$ & - & $(15)$ & $\begin{array}{l}\text { Search, context } \\
\text { memory } \\
(15 \text { min })\end{array}$ & $\underset{\text { impaired }}{\mathrm{SG}}$ & $\begin{array}{c}\mathrm{SG} \\
\text { impaired }\end{array}$ & - & - \\
\hline Hidalgo $^{16}(1)$ & 27 & 25 & $56-76(-)$ & $\begin{array}{l}30 \text { color } \\
\text { pictures }\end{array}$ & $24 \mathrm{~h}$ & TSST & 4-6 p.m. & $\begin{array}{l}\text { Low } \\
(25)\end{array}$ & $\begin{array}{l}\text { No } \\
(-)\end{array}$ & $\begin{array}{l}\text { Free recall } \\
(25 \mathrm{~min})\end{array}$ & Same & Same & - & - \\
\hline Hidalgo $^{16}(2)$ & 27 & 25 & $56-76(-)$ & $\begin{array}{l}30 \text { color } \\
\text { pictures }\end{array}$ & $24 \mathrm{~h}$ & TSST & 4-6 p.m. & $\begin{array}{l}\text { Yes } \\
(25)\end{array}$ & $\begin{array}{l}\text { No } \\
(-)\end{array}$ & $\begin{array}{l}\text { Recognition } \\
(25 \mathrm{~min})\end{array}$ & Same & Same & - & - \\
\hline Hidalgo $^{16}(3)$ & 26 & 24 & $18-27(-)$ & $\begin{array}{l}30 \text { color } \\
\text { pictures }\end{array}$ & $24 \mathrm{~h}$ & TSST & 4-6 p.m. & $\begin{array}{l}\text { High } \\
(25)\end{array}$ & $\begin{array}{l}\text { High } \\
(25)\end{array}$ & $\begin{array}{l}\text { Free recall } \\
(25 \mathrm{~min})\end{array}$ & $\underset{\text { impaired }}{\text { SG }}$ & Same & - & - \\
\hline Hidalgo $^{16}(4)$ & 26 & 24 & $18-27(-)$ & $\begin{array}{l}30 \text { color } \\
\text { pictures }\end{array}$ & $24 \mathrm{~h}$ & TSST & 4-6 p.m. & $\begin{array}{l}\text { Yes } \\
(25)\end{array}$ & $\begin{array}{l}\text { Yes } \\
(25)\end{array}$ & $\begin{array}{l}\text { Recognition } \\
(25 \mathrm{~min})\end{array}$ & Same & Same & - & - \\
\hline Hupbach ${ }^{10}$ & 37 & 38 & $18-35(-)$ & $\begin{array}{l}\text { Neutral text } \\
\text { with } 30 \text { ideas }\end{array}$ & $24 \mathrm{~h}$ & $\begin{array}{l}\text { CPT }\left(0-2{ }^{\circ} \mathrm{C}\right. \\
\text { for } 2 \mathrm{~min})\end{array}$ & $\begin{array}{l}11 \text { a.m.- } \\
\text { 4:30 p.m. }\end{array}$ & $\begin{array}{l}\text { Yes } \\
(20)\end{array}$ & $\begin{array}{l}\text { No } \\
(-)\end{array}$ & $\begin{array}{c}\text { Free recall } \\
\text { (immediate) }\end{array}$ & $\begin{array}{c}\text { SG } \\
\text { enhanced }\end{array}$ & Same & - & - \\
\hline Larrosa $^{28}$ & 6 & 12 & $18-40(-)$ & $\begin{array}{l}5 \text { pairs of } \\
\text { nonsense } \\
\text { syllables }\end{array}$ & 3 days & $\begin{array}{l}\text { CPT }\left(0-4{ }^{\circ} \mathrm{C}\right. \\
\text { for } 1 \mathrm{~min})\end{array}$ & $\begin{array}{c}11 \text { a.m. }-5 \\
\text { p.m. }\end{array}$ & $\begin{array}{l}\text { Yes } \\
(20)\end{array}$ & $\begin{array}{l}\text { Yes } \\
(20)\end{array}$ & $\begin{array}{l}\text { Cued recall } \\
(20 \mathrm{~min})\end{array}$ & $\underset{\text { impaired }}{\mathrm{SG}}$ & $\underset{\text { impaired }}{\mathrm{SG}}$ & - & - \\
\hline Pulopulos ${ }^{17}(1)$ & 38 & 38 & $56-76(-)$ & $\begin{array}{l}30 \text { color } \\
\text { pictures }\end{array}$ & $24 \mathrm{~h}$ & TSST & 4-6 p.m. & $\begin{array}{l}\text { High } \\
(25)\end{array}$ & $\begin{array}{l}\text { Low } \\
\text { (25) }\end{array}$ & $\begin{array}{l}\text { Free recall } \\
(25 \mathrm{~min})\end{array}$ & Same & Same & - & - \\
\hline Pulopulos ${ }^{17}$ (2) & 38 & 38 & $56-76(-)$ & $\begin{array}{l}15 \text { neutral } \\
\text { words }\end{array}$ & $24 \mathrm{~h}$ & TSST & 4-6 p.m. & $\begin{array}{l}\text { High } \\
(25)\end{array}$ & $\begin{array}{l}\text { Low } \\
\text { (25) }\end{array}$ & $\begin{array}{l}\text { Free recall } \\
(40 \mathrm{~min})\end{array}$ & Same & Same & - & - \\
\hline Pulopulos ${ }^{17}(3)$ & 38 & 38 & $56-76(-)$ & 2 stories (42 & $24 \mathrm{~h}$ & TSST & 4-6 p.m. & $\begin{array}{l}\text { High } \\
(25)\end{array}$ & $\begin{array}{l}\text { Low } \\
\text { (25) }\end{array}$ & $\begin{array}{l}\text { Free recall } \\
(45 \mathrm{~min})\end{array}$ & Same & Same & - & - \\
\hline Schonfeld ${ }^{14}(1)$ & 36 & 36 & $-(23.2)$ & $\begin{array}{l}50 \text { nouns } \\
\text { and } 50 \\
\text { pictures }\end{array}$ & $24 \mathrm{~h}$ & $\begin{array}{l}\text { TSST (core } \\
\text { items) }\end{array}$ & $\begin{array}{l}1-6: 30 \\
\text { p.m. }\end{array}$ & $\begin{array}{l}\text { High } \\
(25)\end{array}$ & $\begin{array}{l}\text { Low } \\
(25)\end{array}$ & $\begin{array}{c}\text { Free recall } \\
\text { (immediate) }\end{array}$ & Same & Same & $\begin{array}{l}\text { RD/LD } \\
\text { impaired }^{+}\end{array}$ & $\begin{array}{c}\text { RD/LD } \\
\text { impaired }^{+}\end{array}$ \\
\hline Schonfeld ${ }^{14}(2)$ & 36 & 36 & $-(23.2)$ & $\begin{array}{l}50 \text { nouns } \\
\text { and } 50 \\
\text { pictures }\end{array}$ & $24 \mathrm{~h}$ & $\begin{array}{l}\text { TSST (core } \\
\text { items) }\end{array}$ & $\begin{array}{l}1-6: 30 \\
\text { p.m. }\end{array}$ & $\begin{array}{l}\text { High } \\
(25)\end{array}$ & $\begin{array}{l}\text { Low } \\
(25)\end{array}$ & $\begin{array}{c}\text { Free recall } \\
(25 \mathrm{~min})\end{array}$ & Same & Same & $\begin{array}{c}\mathrm{RD} / \mathrm{LD} \\
\text { impaired }^{+}\end{array}$ & $\begin{array}{c}\mathrm{RD} / \mathrm{LD} \\
\text { impaired }^{+}\end{array}$ \\
\hline Schoofs \& Wolf ${ }^{18}$ & - & 36 & $-(24.7)$ & 30 nouns & $24 \mathrm{~h}$ & TSST & $\begin{array}{c}10-11 \\
\text { a.m. }\end{array}$ & $-(-)$ & $\begin{array}{l}\text { Low } \\
(10)\end{array}$ & $\begin{array}{c}\text { Free recall } \\
(25 \mathrm{~min})\end{array}$ & - & Same & - & Same \\
\hline Schwabe $^{15}(1)$ & 20 & 20 & $-(23.6)$ & $\begin{array}{c}2 \text { lists of } 60 \\
\text { nouns }\end{array}$ & $24 \mathrm{~h}$ & $\begin{array}{l}\text { SECPT ( }(0-2 \\
{ }^{\circ} \mathrm{C} \text { for } 3 \\
\text { min) }\end{array}$ & 1-6 p.m. & $\begin{array}{l}\text { No } \\
(-)\end{array}$ & $\begin{array}{l}\text { No } \\
(-)\end{array}$ & $\begin{array}{l}\text { Recognition } \\
\text { (immediate) }\end{array}$ & Same & Same & - & - \\
\hline Schwabe $^{15}(2)$ & 20 & 20 & $-(23.6)$ & $\begin{array}{l}2 \text { lists of } 60 \\
\text { nouns }\end{array}$ & $24 \mathrm{~h}$ & $\begin{array}{l}\text { SECPT ( } 0-2 \\
{ }^{\circ} \mathrm{C} \text { for } 3 \\
\text { min) }\end{array}$ & 1-6 p.m. & $\begin{array}{l}\text { Yes } \\
(25)\end{array}$ & $\begin{array}{l}\text { Yes } \\
(25)\end{array}$ & $\begin{array}{l}\text { Recognition } \\
\text { (25 min) }\end{array}$ & $\underset{\text { impaired }^{+}}{\mathrm{SG}}$ & $\underset{\text { impaired }^{\ddagger}}{\mathrm{SG}}$ & - & - \\
\hline Schwabe $^{15}(3)$ & 20 & 20 & $-(23.6)$ & $\begin{array}{l}2 \text { lists of } 60 \\
\text { nouns }\end{array}$ & $24 \mathrm{~h}$ & $\begin{array}{l}\text { SECPT ( } 0-2 \\
{ }^{\circ} \mathrm{C} \text { for } 3 \\
\text { min) }\end{array}$ & 1-6 p.m. & $\begin{array}{l}\text { No } \\
(-)\end{array}$ & $\begin{array}{l}\text { No } \\
(-)\end{array}$ & $\begin{array}{l}\text { Recognition } \\
\text { (90 min) }\end{array}$ & $\underset{\text { impaired }}{\mathrm{SG}}$ & $\underset{\text { impaired }}{\mathrm{SG}}$ & - & - \\
\hline Smeets $^{23}(1)$ & 17 & 21 & $\begin{array}{c}18- \\
25(19.9)\end{array}$ & 30 words & $24 \mathrm{~h}$ & $\begin{array}{l}\text { SECPT (0-2 } \\
{ }^{\circ} \mathrm{C} \text { for } 3 \\
\text { min) }\end{array}$ & 9-11 a.m. & $\begin{array}{l}\text { Yes } \\
(20)\end{array}$ & $\begin{array}{l}\text { Yes } \\
(20)\end{array}$ & $\begin{array}{l}\text { Free recall } \\
(15-25 \mathrm{~min})\end{array}$ & $\begin{array}{c}\text { SG } \\
\text { impaired }\end{array}$ & $\underset{\text { impaired }}{S G}$ & - & - \\
\hline Smeets $^{23}(2)$ & 17 & 21 & $\begin{array}{l}18-25 \\
(19.9)\end{array}$ & 30 words & $24 \mathrm{~h}$ & $\begin{array}{l}\text { SECPT ( } 0-2 \\
{ }^{\circ} \mathrm{C} \text { for } 3 \\
\text { min) }\end{array}$ & 2-6 p.m. & $\begin{array}{l}\text { Yes } \\
(20)\end{array}$ & $\begin{array}{l}\text { Yes } \\
(20)\end{array}$ & $\begin{array}{l}\text { Free recall } \\
(15-25 \mathrm{~min})\end{array}$ & $\begin{array}{c}\text { SG } \\
\text { impaired }\end{array}$ & $\underset{\text { impaired }}{S G}$ & - & - \\
\hline Smith $^{29}(1)$ & 48 & 72 & $-(20.1)$ & $\begin{array}{l}30 \text { nouns } \\
\text { and } 30 \\
\text { images }\end{array}$ & $24 \mathrm{~h}$ & TSST-G & $\begin{array}{c}\text { 3:30-5:30 } \\
\text { p.m. }\end{array}$ & $\begin{array}{l}\text { Yes } \\
(-)\end{array}$ & $\begin{array}{l}\text { Yes } \\
(-)\end{array}$ & $\begin{array}{l}\text { Free recall } \\
\text { (immediate) }\end{array}$ & Same & Same & - & - \\
\hline Smith $^{29}(2)$ & 48 & 72 & $-(20.1)$ & $\begin{array}{l}30 \text { nouns } \\
\text { and } 30 \\
\text { images }\end{array}$ & $24 \mathrm{~h}$ & TSST-G & $\begin{array}{c}\text { 3:30-5:30 } \\
\text { p.m. }\end{array}$ & $\begin{array}{l}\text { Yes } \\
(-)\end{array}$ & $\begin{array}{l}\text { Yes } \\
(-)\end{array}$ & $\begin{array}{l}\text { Free recall } \\
(20 \mathrm{~min})\end{array}$ & $\begin{array}{c}\text { SG } \\
\text { impaired }\end{array}$ & $\underset{\text { impaired }}{\mathrm{SG}}$ & $\begin{array}{l}\text { RP same } \\
\text { as CG }\end{array}$ & $\begin{array}{l}\text { RP same } \\
\text { as CG }\end{array}$ \\
\hline Szollosi ${ }^{30}$ & 28 & 35 & $19-27(-)$ & $\begin{array}{l}40 \text { word } \\
\text { pairs }\end{array}$ & 7 days & TSST & 4-8 p.m. & $\begin{array}{l}\text { Yes } \\
(-)\end{array}$ & $\begin{array}{l}\text { Yes } \\
(-)\end{array}$ & $\begin{array}{l}\text { Cued recall } \\
(30 \mathrm{~min})\end{array}$ & Same & Same & $\begin{array}{c}\mathrm{RD} \\
\text { impaired }\end{array}$ & $\begin{array}{c}\mathrm{RD} \\
\text { impaired }\end{array}$ \\
\hline Zoladz $^{11}(1)$ & 38 & 55 & - (19.45) & 42 words & $5 \mathrm{~min}$ & $\begin{array}{l}\text { CPT }\left(0-2{ }^{\circ} \mathrm{C}\right. \\
\text { for } 3 \mathrm{~min})\end{array}$ & $\begin{array}{l}\text { Noon-6 } \\
\text { p.m. }\end{array}$ & $\begin{array}{l}\text { Low } \\
\text { (22) }\end{array}$ & $\begin{array}{l}\text { Low } \\
\text { (22) }\end{array}$ & $\begin{array}{c}\text { Free recall } \\
\text { (immediate) }\end{array}$ & Same & Same & $\begin{array}{c}\mathrm{RD} \\
\text { enhanced }\end{array}$ & Same \\
\hline Zoladz $^{11}(2)$ & 38 & 55 & - (19.45) & 42 words & $24 \mathrm{~h}$ & $\begin{array}{l}\text { CPT }\left(0-2^{\circ} \mathrm{C}\right. \\
\text { for } 3 \mathrm{~min})\end{array}$ & $\begin{array}{l}\text { Noon-6 } \\
\text { p.m. }\end{array}$ & $\begin{array}{l}\text { Low } \\
\text { (22) }\end{array}$ & $\begin{array}{l}\text { Low } \\
(22)\end{array}$ & $\begin{array}{l}\text { Recognition } \\
\text { (22 min) }\end{array}$ & Same & Same & $\begin{array}{c}\text { NR } \\
\text { impaired }\end{array}$ & Same \\
\hline
\end{tabular}

Numbers in parentheses next to author name represent an experimental condition or group in the corresponding study.

- = not available; $C G=$ control group; $C P T=$ Cold-Pressor Test; $F=$ female; $L D=$ participants with low diastolic blood pressure; $M=$ male; $\mathrm{NR}=$ cortisol nonresponders; RD = cortisol responders; RP = retrieval practice condition; SECPT = Socially Evaluated Cold-Pressor Test; SG = stress group; TSST = Trier Social Stress Test; TSST-G = TSST group version.

* After controlling for changes in energetic and tense arousal, the association between increase in salivary cortisol and memory retrieval was no longer significant.

+ Only for negative items.

₹ Marginally significant. 
and their memory is less impaired than men's, despite reliable cortisol increase following stress induction via TSST (the results reported by Schoofs \& Wolf ${ }^{18}$ contrast with earlier results from the same laboratory ${ }^{33}$ ).

Table 2 - Summary of study characteristics included in the systematic review

\begin{tabular}{|c|c|}
\hline Variable & $\begin{array}{c}\text { No. } \\
\text { experiments }\end{array}$ \\
\hline \multicolumn{2}{|l|}{ Memory test type } \\
\hline Recognition & 6 \\
\hline Free recall & 15 \\
\hline Cued recall & 2 \\
\hline Search task & 2 \\
\hline \multicolumn{2}{|c|}{ Participants' characteristics } \\
\hline Young men & 19 \\
\hline Young women & 19 \\
\hline Old men & 5 \\
\hline Old women & 5 \\
\hline \multicolumn{2}{|l|}{ Time of day } \\
\hline Morning & 2 \\
\hline Lunch time & 2 \\
\hline Early afternoon & 1 \\
\hline Late afternoon & 11 \\
\hline Whole afternoon & 9 \\
\hline \multicolumn{2}{|l|}{ Retention interval } \\
\hline $135 \mathrm{~min}$ & 1 \\
\hline $24 \mathrm{~h}$ & 21 \\
\hline 3 days & 1 \\
\hline 7 days & 1 \\
\hline 5 months & 1 \\
\hline \multicolumn{2}{|l|}{ Stimulus type } \\
\hline Pictures & 8 \\
\hline Words & 13 \\
\hline Non-word syllable & 1 \\
\hline Text passage & 2 \\
\hline Search task & 2 \\
\hline Word pairs & 1 \\
\hline \multicolumn{2}{|c|}{ Stress-induction protocols (stressors) } \\
\hline TSST & 14 \\
\hline СРT & 6 \\
\hline SECPT & 5 \\
\hline \multicolumn{2}{|c|}{ Stressor onset (min before criterial test) } \\
\hline Immediate & 5 \\
\hline 15 to $25 \mathrm{~min}$ & 15 \\
\hline 30 to $45 \mathrm{~min}$ & 4 \\
\hline $90 \mathrm{~min}$ & 1 \\
\hline
\end{tabular}

TSST = Trier Social Stress Test; CPT = Cold-Pressor Test; SECPT = Socially Evaluated Cold-Pressor Test.

\section{Time of day}

Cortisol levels are usually higher in the morning when compared to the afternoon. Most studies held the retrieval session in the afternoon, with only two studies having taken place in the morning. ${ }^{18,23}$ Smeets $^{23}$ specifically assessed the role of time of day on stressinduced retrieval impairment by dividing participants into two groups, one with the session in the morning and the other in the afternoon. He found no difference in memory performance between the groups (morning or afternoon), suggesting that stress-induced retrieval deficits occur independently of time of day and that retrieval deficits are more related to the proportion of the increase in cortisol levels than to the absolute cortisol concentrations.

\section{Discussion}

We reviewed studies assessing the impact of stressinduced cortisol increases on memory retrieval. In particular, we looked at differences in stressors, stressretrieval delays, time of day of stress induction, sex and age of participants. The reviewed studies indicate that acute stress does impair retrieval, particularly when induced with the TSST in the afternoon up to 45 minutes before the onset of the final (recall) test in healthy young men.

The stress protocols used in the reviewed studies (CPT, SECPT, TSST) induced reliable increases in cortisol concentrations in both young men and women. These stressors, however, were less efficient with older participants of both sexes. ${ }^{16}$ The reason why older participants are less affected by these stressors is still an open question. One possibility relates to the fact that cortisol receptors in older participants are less densely distributed and less responsive in memory-relevant areas (hippocampus and prefrontal cortex) than in younger participants. ${ }^{34,35}$ This could partly account for the lower stress-induced memory impairments observed so far in older populations. Another possibility is that older participants are more habituated than younger participants to psychosocial stress. This possibility, however, is at odds with findings that older participants under stress (e.g., TSST) release more salivary alpha amylase, a SNS activity marker, than younger participants. ${ }^{21}$ In addition, older participants may experience higher levels of subjective stress than younger participants, possibly due to the unfamiliarity and unpredictability of the testing environment, which is more familiar to younger university students. ${ }^{9}$ Thus, it is not yet clear why stress-induced memory effects differ across age groups. Longitudinal studies (as 
opposed to the more common cross-sectional studies) coupled with novel stressor combinations (TSST plus SECPT, as proposed by Boyle et al. ${ }^{36}$ ) may induce more reliable cortisol increases in older participants and thus facilitate the assessment of cortisol-related effects in memory in this population.

Some stress-inducing protocols are more effective at eliciting HPA-axis responses than others. The TSST induces stronger cortisol and subjective stress responses than the CPT. ${ }^{37}$ In addition, the TSST induces more sustained stress responses than the SECPT. ${ }^{38}$ The subjective and SNS components of the stress response in the SECPT return to baseline by the time cortisol reaches peak levels. Adding the fact that the TSST has been studied for longer than the SECPT, it is understandable that most studies designed to assess the role of stress on memory chose to induce stress via the TSST. However, the SECPT has some attractive features, such as shorter application time (less than 3 minutes vs. 15 minutes in the TSST) and the presence of only one committee member (vs. three in the TSST). Similarly to the TSST, the SECPT has also been extended to group application and has been proven resistant to habituation effects. ${ }^{39}$ Thus, future studies using SECPT or hybrid TSST-SECPT protocols ${ }^{36}$ may help clarify the impact of HPA axis (and concurrent SNS) activation on memory retrieval.

Sex differences on memory performance were not evident in the reviewed studies. Women usually outperform men on episodic memory tasks. ${ }^{40,41}$ Despite superior memory, women are not immune to stressrelated retrieval impairment, ${ }^{29}$ particularly if they are in the follicular phase of the menstrual cycle and not taking oral contraceptives. ${ }^{7,21}$ By contrast, women taking contraceptives or in the luteal phase of the menstrual cycle have their memories less affected by surges in cortisol levels. 7,18 The lack of sex differences in stressrelated memory impairments is consistent with the conclusions of a recent, focused review. ${ }^{35}$

Even though stress impairs retrieval, ${ }^{7}$ it does so more strongly for materials that have been encoded via repeated study than for materials that have been encoded via repeated retrieval. ${ }^{29,42}$ The latter strategy (retrieval practice) results in more enduring memories, ${ }^{43}$ even when compared to active encoding strategies. ${ }^{44}$ Because both memory and stress are ubiquitous in learning contexts, ${ }^{45}$ it is important to understand how they interact to improve or impair student outcomes. One direction for future research involves assessing whether the protection against the deleterious effects of stress afforded by retrieval practice is similar to different types of study materials (e.g., easy- vs. hard-to-learn materials), as difficult materials may be particularly affected by stress during high-stakes tests.
One limitation of the present review was its restricted scope. The focus on stress at retrieval was motivated by the ubiquity of stressors during recall tasks (e.g., during exams) and by the promising protective effects of retrieval practice on stress-related memory deficits. The restricted scope of our review, and consequent small number of eligible studies, also prevented us from conducting a quantitative analysis on the studies returned by the search. As the field progresses, future reviews may be able to quantify the individual roles of age, sex, and type of stressor on stress-related memory deficits.

In conclusion, we reviewed research assessing the impact of stress on memory retrieval. The reviewed studies indicate that stress impairs retrieval, particularly when induced with the TSST in healthy young men. These results may inform future research on the impact of stress-induced cortisol increases on memory retrieval.

\section{Acknowledgements}

This work was supported by Coordenação de Aperfeiçoamento de Pessoal de Nível Superior (CAPES).

\section{Disclosure}

No conflicts of interest declared concerning the publication of this article.

\section{References}

1. Schlotz W, Kumsta R, Layes I, Entringer S, Jones A, Wust S. Covariance between psychological and endocrine responses to pharmacological challenge and psychosocial stress: a question of timing. Psychosom Med. 2008;70:787-96.

2. Wirth MM. Hormones, stress, and cognition: the effects of glucocorticoids and oxytocin on memory. Adapt Hum Behav Physiol. 2015;1:177-201.

3. Wolf OT. Stress and memory retrieval: mechanisms and consequences. Curr Opin Behav Sci. 2017;14:40-6.

4. Gagnon SA, Wagner AD. Acute stress and episodic memory retrieval: neurobiological mechanisms and behavioral consequences. Ann N Y Acad Sci. 2016;1369:55-75.

5. Lonergan $M H$, Olivera-Figueroa LA, Pitman RK, Brunet A. Propranolol's effects on the consolidation and reconsolidation of long-term emotional memory in healthy participants: a metaanalysis. J Psychiatry Neurosci. 2013;38:222-31.

6. Schwabe L, Joels M, Roozendaal B, Wolf OT, Oitzl MS. Stress effects on memory: an update and integration. Neurosci Biobehav Rev. 2012;36:1740-9.

7. Shields GS, Sazma MA, McCullough AM, Yonelinas AP. The effects of acute stress on episodic memory: a meta-analysis and integrative review. Psychol Bull. 2017;143:636-75.

8. Roozendaal B, McGaugh JL. Memory modulation. Behav Neurosci. 2011;125:797-824. 
9. Lupien SJ, Maheu F, Tu M, Fiocco A, Schramek TE. The effects of stress and stress hormones on human cognition: implications for the field of brain and cognition. Brain Cogn. 2007;65:209-37.

10. Hupbach A, Fieman R. Moderate stress enhances immediate and delayed retrieval of educationally relevant material in healthy young men. Behav Neurosci. 2012;126:819-25.

11. Zoladz PR, Kalchik AE, Hoffman MM, Aufdenkampe RL, Burke HM, Woelke SA, et al. Brief, pre-retrieval stress differentially influences long-term memory depending on sex and corticosteroid response. Brain Cogn. 2014;85:277-85.

12. Boehringer $A$, Schwabe $L$, Schachinger $H$. A combination of high stress-induced tense and energetic arousal compensates for impairing effects of stress on memory retrieval in men. Stress. 2010;13:444-53.

13. Goldfarb EV, Mendelevich $Y$, Phelps EA. Acute stress timedependently modulates multiple memory systems. J Cogn Neurosci. 2017;29:1877-94.

14. Schonfeld P, Ackermann K, Schwabe L. Remembering under stress: Different roles of autonomic arousal and glucocorticoids in memory retrieval. Psychoneuroendocrinology. 2014;39:249-56.

15. Schwabe L, Wolf OT. Timing matters: Temporal dynamics of stress effects on memory retrieval. Cogn Affect Behav Neurosci. 2014;14:1041-8.

16. Hidalgo V, Pulopulos MM, Puig-Perez S, Espin L, Gomez-Amor J, Salvador A. Acute stress affects free recall and recognition of pictures differently depending on age and sex. Behav Brain Res. 2015;292:393-402.

17. Pulopulos MM, Almela M, Hidalgo V, Villada C, Puig-Perez S, Salvador A. Acute stress does not impair long-term memory retrieval in older people. Neurobiol Learn Mem. 2013;104:1624.

18. Schoofs D, Wolf OT. Stress and memory retrieval in women: no strong impairing effect during the luteal phase. Behav Neurosci. 2009;123:547-54.

19. Lovallo W. The Cold Pressor Test and autonomic function: a review and integration. Psychophysiology. 1975;12:268-82.

20. Kirschbaum C, Pirke KM, Hellhammer DH. The 'Trier Social Stress Test'-- a tool for investigating psychobiological stress responses in a laboratory setting. Neuropsychobiology. 1993;28:76-81.

21. Allen AP, Kennedy PJ, Dockray S, Cryan JF, Dinan TG, Clarke G. The Trier Social Stress Test: Principles and practice. Neurobiol Stress. 2017;6:113-26.

22. Rimmele $U$, Meier F, Lange T, Born J. Suppressing the morning rise in cortisol impairs free recall. Learn Mem. 2010;17:186-90.

23. Smeets T. Acute stress impairs memory retrieval independent of time of day. Psychoneuroendocrinology. 2011;36:495-501.

24. Perlman WR, Webster MJ, Herman MM, Kleinman JE, Weickert CS. Age-related differences in glucocorticoid receptor mRNA levels in the human brain. Neurobiol Aging. 2007;28:447-58.

25. Kirschbaum C, Kudielka BM, Gaab J, Schommer NC, Hellhammer $\mathrm{DH}$. Impact of gender, menstrual cycle phase, and oral contraceptives on the activity of the hypothalamus-pituitaryadrenal axis. Psychosom Med. 1999;61:154-62.

26. Moher D, Liberati A, Tetzlaff J, Altman DG, Group P. Preferred reporting items for systematic reviews and meta-analyses: the PRISMA statement. ] Clin Epidemiol. 2009;62:1006-12.

27. Panic N, Leoncini E, de Belvis G, Ricciardi W, Boccia S. Evaluation of the endorsement of the preferred reporting items for systematic reviews and meta-analysis (PRISMA) statement on the quality of published systematic review and meta-analyses. PloS One. 2013;8:e83138.
28. Larrosa PNF, Ojea A, Ojea I, Molina VA, Zorrilla-Zubilete MA, Delorenzi A. Retrieval under stress decreases the long-term expression of a human declarative memory via reconsolidation. Neurobiol Learn Mem. 2017;142:135-45.

29. Smith AM, Floerke VA, Thomas AK. Retrieval practice protects memory against acute stress. Science. 2016;354:1046-8.

30. Szőllősi Á, Keresztes A, Novák B, Szászi B, Kéri S, Racsmány $M$. The testing effect is preserved in stressful final testing environment. Appl Cogn Psychol. 2017;31:615-22.

31. Schwabe L, Haddad L, Schachinger H. HPA axis activation by a socially evaluated cold-pressor test. Psychoneuroendocrinology. 2008;33:890-5.

32. Tollenaar MS, Elzinga BM, Spinhoven $P$, Everaerd WA. The effects of cortisol increase on long-term memory retrieval during and after acute psychosocial stress. Acta Psychol. 2008;127:542-52.

33. Kuhlmann $S$, Piel $M$, Wolf OT. Impaired memory retrieval after psychosocial stress in healthy young men. J Neurosci. 2005; 25:2977-82

34. Heffelfinger AK, Newcomer JW. Glucocorticoid effects on memory function over the human life span. Dev Psychopathol. 2001;13:491-513.

35. Hidalgo V, Pulopulos MM, Salvador A. Acute psychosocial stress effects on memory performance: Relevance of age and sex. Neurobiol Learn Mem. 2019;157:48-60.

36. Boyle NB, Lawton C, Arkbage K, West SG, Thorell L, Hofman D, et al. Stress responses to repeated exposure to a combined physical and social evaluative laboratory stressor in young healthy males. Psychoneuroendocrinology. 2016;63:119-27.

37. Skoluda N, Strahler J, Schlotz W, Niederberger L, Marques S, Fischer $\mathrm{S}$, et al. Intra-individual psychological and physiological responses to acute laboratory stressors of different intensity. Psychoneuroendocrinology. 2015;51:227-36.

38. Giles GE, Mahoney CR, Brunye $T$, Taylor HA, Kanarek RB. Stress effects on mood, HPA axis, and autonomic response: comparison of three psychosocial stress paradigms. PloS One. 2014; 9:e113618.

39. Minkley N, Schroder TP, Wolf OT, Kirchner WH. The socially evaluated cold-pressor test (SECPT) for groups: effects of repeated administration of a combined physiological and psychological stressor. Psychoneuroendocrinology. 2014;45:119-27.

40. Herlitz A, Nilsson LG, Backman L. Gender differences in episodic memory. Mem Cognit. 1997;25:801-11.

41. Herlitz A, Rehnman J. Sex differences in episodic memory. Curr Dir Psychol Sci. 2008;17:52-6.

42. Smith AM, Thomas AK. Reducing the consequences of acute stress on memory retrieval. J Appl Res Mem Cogn. 2018;7:21929.

43. Karpicke JD, Roediger HL 3rd. The critical importance of retrieval for learning. Science. 2008;319:966-8.

44. Karpicke JD, Blunt JR. Retrieval practice produces more learning than elaborative studying with concept mapping. Science. 2011;331:772-5.

45. Vogel S, Schwabe L. Learning and memory under stress: implications for the classroom. NPJ Sci Learn. 2016;1:16011.

\section{Correspondence:}

Cadu Klier

Campus Darcy Ribeiro, ICC Sul, Sala ASS-012/5

70910-900 - Brasília, DF - Brazil

Tel.: +5561999442999

E-mail: caduklier@gmail.com 